\title{
Pricing insurance policies with offsetting relationship - ERRATUM
}

\section{Hamza Hanbali}

DOI: https://doi.org/10.1017/S1748499521000208, Published online by Cambridge University Press, 17 September 2021

The publisher apologises that upon publication of this article the authors name was swapped around, presenting the surname as their first.

The authors correct name is Hamza Hanbali.

The online version of this article has been updated.

\section{Reference}

Hanbali, H. (2021). Pricing insurance policies with offsetting relationship. Annals of Actuarial Science, 1-27. doi: $10.1017 /$ S1748499521000208 